\title{
Genomic Encyclopedia of Type Strains, Phase I: The one thousand microbial genomes (KMG-I) project
}

\author{
Nikos C. Kyrpides ${ }^{1}$, Tanja Woyke ${ }^{1}$, Jonathan A. Eisen ${ }^{2}$, George Garrity ${ }^{3,4}$, Timothy G. \\ Lilburn $^{5}$, Brian J. Beck ${ }^{5}$, William B. Whitman ${ }^{6}$, Phil Hugenholtz ${ }^{7}$, and Hans-Peter Klenk ${ }^{8}$ \\ ${ }^{1}$ DOE Joint Genome Institute, Walnut Creek, CA \\ ${ }^{2}$ University of California, Davis, CA \\ ${ }^{3}$ Department of Microbiology and Molecular Genetics, Michigan State University, East \\ Lansing, Michigan, USA \\ ${ }^{4}$ NamesforLife, LLC, East Lansing, MI, USA \\ ${ }^{5}$ American Type Culture Collection, Manassas, VA \\ ${ }^{6}$ Department of Microbiology, University of Georgia, Athens, GA \\ ${ }^{7}$ Australian Centre for Ecogenomics, The University of Queensland, Brisbane QLD 4072, \\ Australia \\ ${ }^{8}$ Leibniz Institute DSMZ - German Collection of Microorganisms and Cell Cultures, \\ Braunschweig, Germany \\ Correspondence: Nikos C. Kyrpides (nckyrpides@lbl.gov)
}

\begin{abstract}
The Genomic Encyclopedia of Bacteria and Archaea (GEBA) project was launched by the JGI in 2007 as a pilot project with the objective of sequencing 250 bacterial and archaeal genomes. The two major goals of that project were (a) to test the hypothesis that there are many benefits to the use the phylogenetic diversity of organisms in the tree of life as a primary criterion for generating their genome sequence and (b) to develop the necessary framework, technology and organization for large-scale sequencing of microbial isolate genomes. While the GEBA pilot project has not yet been entirely completed, both of the original goals have already been successfully accomplished, leading the way for the next phase of the project.

Here we propose taking the GEBA project to the next level, by generating high quality draft genomes for 1,000 bacterial and archaeal strains. This represents a combined 16-fold increase in both scale and speed as compared to the GEBA pilot project (250 isolate genomes in 4+ years). We will follow a similar approach for organism selection and sequencing prioritization as was done for the GEBA pilot project (i.e. phylogenetic novelty, availability and growth of cultures of type strains and DNA extraction capability), focusing on type strains as this ensures reproducibility of our results and provides the strongest linkage between genome sequences and other knowledge about each strain. In turn, this project will constitute a pilot phase of a larger effort that will target the genome sequences of all available type strains of the Bacteria and Archaea.
\end{abstract}

\section{Introduction}

\section{The importance of the research}

In June 2011, there were approximately 9,000 bacterial and archaeal species with validly published names, and with rare exceptions there was a viable sample of the designated type strain available from one or more service culture collections. Although commonly misconstrued as the archetypal representative of a species, type strains are instead live specimens that serve as a fixed reference points to which bacterial and archaeal names are attached. These names (and by reference the type strains) provide an entry into the literature, databases, and our knowledge of microbial diversity. Thus, type strains play a crucial role in defining the phylogenomic and taxonomic space of Bacteria and Archaea. By definition, type strains are descendants of the original isolates that were defined in species and subspecies descriptions, as requested by the Bacteriological Code [1] and exhibit all of the relevant phenotypic and genotypic properties cited in the original published taxonomic circumscriptions. Except in cases such as some symbionts and other 
non-cultivable species defined prior to the 1999 revisions to the rules [2-4], virtually all type strains are available in pure axenic cultures.

Although the Genomic Encyclopedia of Bacteria and Archaea (GEBA) pilot project [5] provided a significant boost in the number of sequenced type strains, upon its completion only $13 \%$ of the total number of available type strains were sequenced [6]. From that fraction, only $7 \%$ had sequence data available as complete or draft sequences (Figure $1)$. Given that the number of newly described species and subspecies (along with the relevant type strains) increases by about 600 per year, the overall coverage of taxonomic types by genome sequencing projects has remained stable during the last several years even as more sequences have been completed.

In addition, the number of genome projects for the currently defined type strains represent about $13 \%$ of the overall number of registered genome sequencing projects for bacterial and archaeal strains [6].

Genomic sequencing of all of the type strains is an important goal. This proposal represents the first phase of this effort, setting the goal of sequencing the first 1,000 of those remaining type strains. In the GEBA pilot project [5] we asked: How much of the known phylogenetic diversity of Bacteria and Archaea has been sampled? Here in KMG1, we extend that question to: How quickly, thoroughly and efficiently can we fill in the gaps and extend our knowledge of the cultivated Bacterial and Archaeal species?

Much of the scientific value and anticipated advantages of such a large effort $[7,8]$ has already been provided from the much smaller scale of the GEBA pilot project [5]. We have addressed this by estimating the diversity of all the cultured species of Bacteria and Archaea (based on the availability of their 16S rRNA sequences) and quantified how much of that diversity has already been sampled.

As shown on Figure 2, with the list of the 1,000 type strains we propose to sequence here, we aim to cover over $40 \%$ of the phylogenetic diversity of cultured members of the Bacteria and Archaea (quantified as the overall distance on the $16 \mathrm{~S}$ rRNA tree). In fact with this proposed list, we will nearly double the currently available phylogenetic diversity of the type strains with finished and ongoing genome sequencing projects.

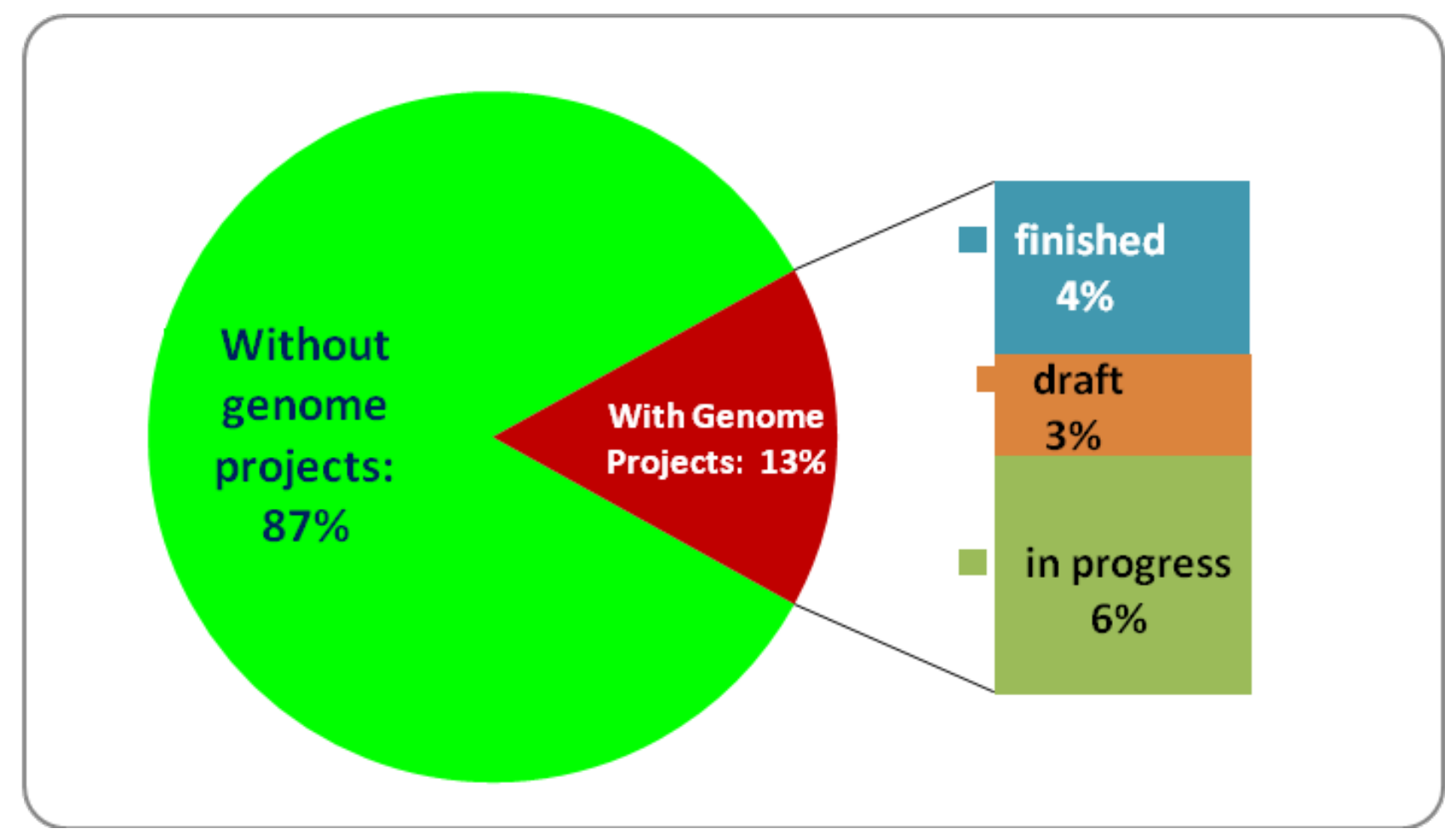

Figure 1. Genome project coverage of bacterial and archaeal type strains as of June 2011. From a total of approximately 9,000 bacterial and archaeal type strains, 1219 (13\%) (non-redundant) have a publicly known genome project. 


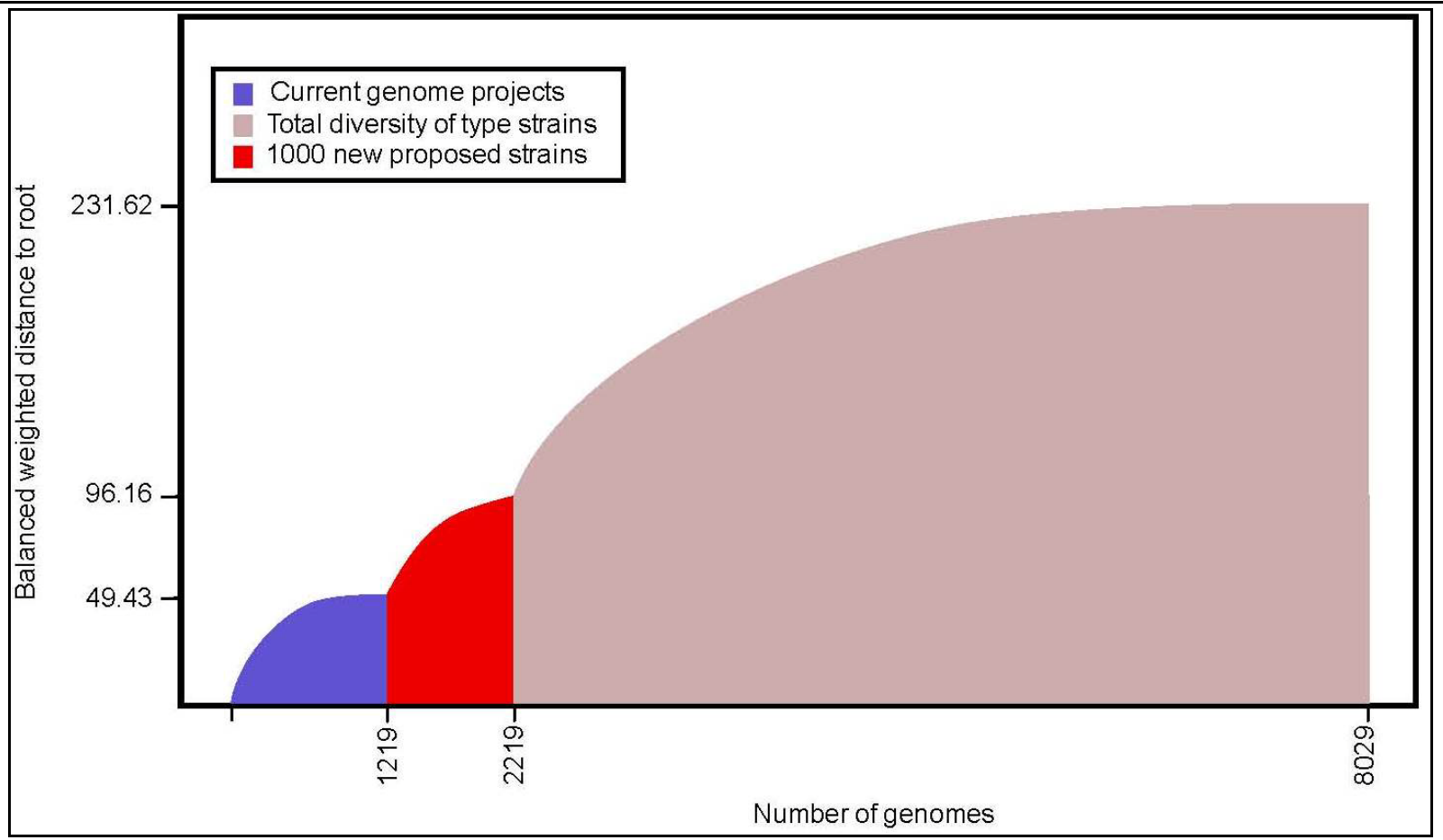

Figure 2. Phylogenetic diversity of the type strains of Bacteria and Archaea based on the SSU rRNA genes as of June 2011. Blue: phylogenetic diversity of current complete and ongoing genome projects from 1,219 type strains (GOLD 1/2011); Red: of the 1,000 type strains proposed to be sequenced here; Pink: phylogenetic diversity of all type strains avai lable at the Living Tree Project (LTP) [9]. All our calculations are based on the LTP tree from September 2010 (latest version) * which contains 8,029 of the about 9,000 type strains.

It should be noted here that while the current list of the type strains with completed and ongoing genome projects (blue color on Fig. 2) is about 1,200, there has not been a systematic effort to sequence type strains, and these represent on a small fraction of the over 7,700 genome projects completed in well over 15 years of microbial genome sequencing. Since a list of 1,000 strains would be too long to list here, we have attached the complete list on a separate file, and we present here only a summary table of the number of organisms we intend to cover per phylum (Table 1).

Totals are based on an export of the Bacterial and Archaeal taxonomic and nomenclatural events in the NamesforLife Database on May 29, 2011. There are 33 named phyla that are currently in common usage to which the validly named species and subspecies were mapped. Percent synonyms were determined based on the number of recorded assertions of homotypic synonymy, new combinations and coordinate status species and subspecies. Heterotypic synonyms are not included in this number as they have separate type strains. Genome sequences are based on those that are declared as types from the GOLD (5/28/2011). Cyanobacteria species are based on those species described in
Bergey's Manual of Systematic Bacteriology, Vol 1., $2^{\text {nd }}$ Ed. 2001 and represent the dominant morphotypes. Non-redundant non-type strains, bearing validly published names, for which types have yet to be sequenced are added to the table to minimize potential for overlap.

\section{Project design}

(a) Selection of target organisms: Target organisms were selected based on a score that measures the relative contribution of each species to the total phylogenetic diversity (PD), as inferred from a phylogenetic tree with computed branch lengths [10]. The underlying phylogenetic tree was the one from the All-Species-Living-Tree-Project in the latest available version from 9/2010, comprising 8,029 leaves (species/subspecies). Species with ongoing or completed genome sequencing projects registered in GOLD (1/2011) were excluded and the highest scoring (PD) species were screened for growth conditions that allow the production of sufficient cell past for DNA extraction. One thousand prime targets were selected and augmented by an additional 685 to serve as a back-up in event of failure to produce sufficient DNA or the inevitabil- 
Kyrpides et al.

ity that another research group may have begun sequencing the same organism.

(b) Organism growth and nucleic acid isolation: All of the proposed strains will be grown at the DSMZ [http://www.dsmz.de/] and ATCC [http://www.atcc.org/] culture collection centers. Both bioresource centers have extensive experience in growing microbial organisms under a wide range of conditions including extremely high temperatures or salt concentration. The new sequencing technologies that will be employed for this project, coupled with an understanding that completely sequenced genomes are not necessarily required to achieve our goals, means that significantly less DNA is needed per individual organism. The current sequencing protocol requires as little as 10 micrograms of DNA for high quality draft genome sequencing, which will greatly facilitate the progress of this project. RNA isolation under a single steady state condition will also be performed at DSMZ.

(c) Sequencing, data QC and assembly: The combination of high throughput sequencing based on the HiSeq Illumina technology, together with our ability to multiply individual channels per flowcell, will allow us to complete this project at a low per unit cost $(<\$ 100$ per genome, unburdened cost). The current plan will be to pool 48-96 microbes per HiSeq channel assuming no major changes in sequencing throughput. This will provide higher than $100 \times$ coverage per microbial genome, and will bring down the total sequencing allocation requested for the 1,000 genomes to a total of 1.5-3 runs. Continued improvements and automation of the JGI sequence QC pipeline ("rolling QC") will enable a largely hands-off approach for the quality assessment and assembly of the sequence data. Although we expect that minor manual intervention will be needed at this stage. Concurrent improvements in bioinformatics and computational power will allow us to assemble most targeted genomes into well under 100 major contigs.

(d) Annotation and comparative analysis: The microbial genome annotation pipeline at the JGI has been scaled to handle hundreds of microbial genomes per month $[9,11,12]$.

(e) Publication of analyzed genomes: Although not all of the draft genomes we propose to generate will be of publication quality, we still plan to publish as many as possible in Standards in Genomic Sciences $[13,14]$. We will use a software pipeline from the GEBA pilot project for accelerated generation of standardized manuscript text that allows (i) automatized collection of sequences and metadata for incorporation in tables and/or text of the manuscripts, (ii) 16S rRNA sequence comparison with the Greengenes database to analyze the target occurrence of the target strain in the environment, including a statistical analysis of keywords (iii) $16 \mathrm{~S}$ rRNA-based phylogenetic analysis and generation of publication-quality tree graphics. Data are provided to the authors via a HTML-based template system. More code for the automated generation of phylogenetic and functional analyses will be available by the time of the proposed start date of the project.

III. The scientific questions we expect to answer The derived data will be utilized in several different ways. We already know from projects such as the GEBA pilot project [5] and the Human Microbiome Project (HMP) project [15] that reference genomes provide significant support in the analysis of metagenomic datasets. It is now well-established that the road to success in metagenomics is through microbial genomics. A systematic coverage of the tree of life will further support several ongoing metagenomic studies. KMG is also strongly endorsed by members of the scientific community leading large international metagenomics studies. The discovery of novel protein families, or novel members of already known protein families, and the overall impact on genome annotation has also been previously documented with much smaller scale sequencing efforts.

In addition to the typical ways the data will be analyzed and compared, and beyond the general evolutionary questions that were posed and explored during the GEBA pilot project, we plan to engage the larger scientific community to work with us and lead various types of additional analyses. Specifically, we plan to organize and facilitate the work of the community either around special phylogenetic groups, around special topics of comparative analysis, and around topics of relevance to the DOE mission (bioenergy, bioremediation, carbon sequestration). This will ensure the maximum benefit of the data derived from this project. This will also be coupled with NamesforLife's ongoing efforts to mine the world's patent literature and to make important connections between what is known about microbes in nature and what is known about the practical uses of these organisms. 
The one thousand microbial genomes

Table 1. Summary table for KMG project (including non-redundant non-type strains)

\begin{tabular}{|c|c|c|c|c|c|c|c|}
\hline Phylum & $\begin{array}{r}\text { Type } \\
\text { strains }\end{array}$ & $\begin{array}{l}\text { Species / } \\
\text { subsp. \% }\end{array}$ & Synonyms & $\begin{array}{r}\text { Type Genome } \\
\text { sequences }\end{array}$ & $\begin{array}{r}\text { Non-type Genome } \\
\text { Sequences }\end{array}$ & Coverage & $\begin{array}{r}\text { Type Genomes } \\
\text { proposed }\end{array}$ \\
\hline Crenarchaeota & 57 & 61 & 6.6 & 33 & 0 & 57.9 & - \\
\hline Euryarchaeota & 314 & 388 & 19.1 & 143 & 4 & 45.5 & 9 \\
\hline Thaumarchaeota & 1 & 1 & 0.0 & 1 & 0 & 100.0 & - \\
\hline Aquificae & 29 & 31 & 6.5 & 8 & 0 & 27.6 & 2 \\
\hline Thermotogae & 37 & 38 & 2.6 & 14 & 1 & 37.8 & 13 \\
\hline Thermodesulfobacteria & 7 & 8 & 12.5 & 2 & 1 & 28.6 & 4 \\
\hline Deinococcus-Thermus & 71 & 76 & 6.6 & 19 & 0 & 26.8 & 18 \\
\hline Chrysiogenetes & 4 & 4 & 0.0 & 2 & 0 & 50.0 & - \\
\hline Chloroflexi & 27 & 28 & 3.6 & 11 & 0 & 40.7 & - \\
\hline Nitrospirae & 12 & 12 & 0.0 & 2 & 0 & 16.7 & 3 \\
\hline Deferribacteres & 12 & 12 & 0.0 & 6 & 0 & 50.0 & 1 \\
\hline Cyanobacteria & 88 & 90 & 2.2 & 9 & 2 & 10.2 & - \\
\hline Chlorobi & 16 & 22 & 27.3 & 9 & 0 & 56.3 & - \\
\hline Proteobacteria & 3,541 & 4,323 & 18.1 & 364 & 35 & 10.3 & 385 \\
\hline Firmicutes & 1,875 & 2,263 & 17.1 & 311 & 14 & 16.6 & 285 \\
\hline Tenericutes & 234 & 258 & 9.3 & 25 & 0 & 10.7 & 2 \\
\hline Actinobacteria & 2,439 & 2,953 & 17.4 & 145 & 5 & 5.9 & 129 \\
\hline Planctomycetes & 15 & 19 & 21.1 & 10 & 0 & 66.7 & 2 \\
\hline Chlamydiae & 17 & 20 & 15.0 & 8 & 0 & 47.1 & - \\
\hline Spirochaetes & 112 & 127 & 11.8 & 25 & 0 & 22.3 & 2 \\
\hline Fibrobacteres & 3 & 5 & 40.0 & 1 & 0 & 33.3 & - \\
\hline Acidobacteria & 11 & 11 & 0.0 & 3 & 0 & 27.3 & - \\
\hline Bacteroidetes & 767 & 914 & 16.1 & 131 & 9 & 17.1 & 134 \\
\hline Fusobacteria & 38 & 47 & 19.1 & 12 & 0 & 31.6 & 7 \\
\hline Verrucomicrobia & 35 & 35 & 0.0 & 6 & 2 & 17.1 & 3 \\
\hline Gemmatimonadetes & 1 & 1 & 0.0 & 1 & 0 & 100.0 & - \\
\hline Dictyoglomi & 2 & 3 & 33.3 & 2 & 0 & 100.0 & - \\
\hline Lentisphaerae & 2 & 2 & 0.0 & 1 & 0 & 50.0 & - \\
\hline Synergistetes & 17 & 18 & 5.6 & 10 & 1 & 58.8 & 1 \\
\hline Caldiserica & 1 & 1 & 0.0 & 1 & 0 & 100.0 & - \\
\hline Elusimicrobia & 1 & 1 & 0.0 & 1 & 0 & 100.0 & - \\
\hline Total & 9,786 & 11,772 & 16.8 & 1,316 & 74 & 14.2 & 1,000 \\
\hline
\end{tabular}


Kyrpides et al.

IV. The size and nature of the larger community that will use the data

We plan to immediately release the data to the public through a variety of channels. Because no restrictions will be associated with the release of the data, the community will be able to make immediate and full use of our findings. The grand scale of the project, the lack of focus on specific applications, and the emphasis on microbial diversity and the tree of life, will most certainly generate novel information of broad scientific interest and benefit for the community at large.

The GEBA pilot project has already generated a great deal of interest in the life sciences research community as well as amongst biology educators, based on the fact that these genomes were released to the community without any restrictions.

The GEBA pilot project has also developed new standards in relation to metadata. The genomes already released to Genbank, have some of the most enriched metadata fields available as compared to any other genome sequencing projects. We will continue with this effort, and major emphasis will be given to the curation and public release of the associated metadata for all of these organisms. This will be done in collaboration and coordination with the Genomic Standards Consortium [16], and we will comply with the recently

\section{Acknowledgements}

This work was performed under the auspices of the US Departm ent of Energy's Office of Science, Biological and Environmental Research Program, and by the University

\section{References}

1. Lapage SP, Sneath PHA, Lessel EF, Skerman VDB, Seelig er HPR, Clark WA, eds. (International Code of Nomenclature (1990 Revision). Bacteriological Code. American Society for Microbiology, Washington, D. C.,1992

2. Trüper HG, Euzéby JP. International Code of Nomenclature of Prokaryotes. Appendix 9: Orthography. Int J Syst Evol Microbiol 2009; 59:2107-

2113. PubMed http://dx.doi.org/10.1099/ijs.0.016741-0

3. Tindall BJ, Kämpfer P, Euzéby JP, Oren A. Valid publication of names of prokaryotes according to the rules of nomenclature: past history and current practice. Int J Syst Evol Microbiol 2006; proposed Minimum Information for Genomics Sequences (MIGS) metadata [14,17].

\section{$V$. The relevance of the project to problems of societal importance}

The selection of sequencing targets will not be based on their specific relevance with a scientific mission, per-se. However, as the GEBA pilot project has demonstrated, a phylogeny-based selection process for sequencing will generate tremendous amounts of fundamental knowledge and information that will impact many fields and realms of science, medicine and technology. As an example, we have shown that the phylogenetic basis of genome project selection is increasing the probability of novel protein family discovery [5]. This is certainly expected to lead to the discovery of novel enzymatic activities with direct relevance to bioenergy, biogeochemistry, bioremediation and carbon cycling applications. We have already published a number of papers based on GEBA pilot genomes demonstrating this fact and several more are currently submitted or in preparation [18-20]. A notable example of one of these studies involves the identification of a number of novel cellulases from a halophilic archaeon (not previously suspected to be an organism likely to harbor cellulases), which were followed by experimental verification $[18,19]$.

of California, Lawrence Berkeley National Laboratory under contract No. DE-AC02-05CH11231.

56:2715-2720. PubMed

http://dx.doi.org/10.1099/ijs.0.64780-0

4. Tindall BJ, Garrity GM. Proposals to clarify how type strains are deposited and made available to the scientific community for the purpose of systematic. Int J Syst Evol Microbiol 2008; 58:19871990. PubMed http://dx.doi.org/10.1099/ijs.0.2008/006155-0

5. Wu D, Hug enholtz P, Mavromatis K, Pukall R, Dalin E, Ivanova NN, Kunin V, Goodwin L, Wu $M$, Tindall BJ, et al. A phylog eny-driven genomic encyclopedia of Bacteria and Archaea. Nature 2009; 462:1056-1060. PubMed http://dx.doi.org/10.1038/nature08656 
6. Liolios K, Chen IM, Mavromatis K, Tavernarakis $\mathrm{H}$, Hug enholtz $\mathrm{P}$, Markovitz VM, Kyrpides NC. The Genomes OnLine Database (GOLD) in 2009: status of genomic and metag enomic projects and their associated metadata. Nucleic Acids Res 2010; 38:D346. PubMed http://dx.doi.org/10.1093/nar/gkp848

7. Kyrpides NC. Fifteen Years of Microbial Genomics: Meeting the Challenges and Fulfilling the Dream. Nat Biotechnol 2009; 27:62 7. PubMed http://dx.doi.org/10.1038/nbt.1552

8. P Hug enholtz. Exploring prokaryotic diversity in the genomic era. Genome Biology 2002; 3: reviews0003.1-0003.8.

9. Mavromatis K, Ivanova NN, Chen IM, Szeto E, Markowitz VM, Kyrpides NC. The DOE-JGl Standard Operating Procedure for the Annotations of Microbial Genomes. Stand Genom ic Sci 2009; 1:63-67. PubMed http://dx.doi.org/10.4056/sigs.632

10. Göker M, Klenk HP. Phylog eny-driven targ et selection for large-scale genome-sequencing (and other) projects. Stand Genom ic Sci 2013; 8:360374. PubMed http://dx.doi.org/10.4056/sigs.3446951

11. Pati A, Ivanova N, Mikhailova N, Ovchinikova G, Hooper SD, Lykidis A, Kyrpides NC. GenePRIMP: A Gene Prediction Improvement Pipeline for microbial genomes. Nat Methods $2010 ; 7: 455-457$. PubMed http://dx.doi.org/10.1038/nmeth.1457

12. Markowitz VM, Ivanova NN, Chen IMA, Chu K, Kyrpides NC. IMG ER: a system for microbial genome annotation expert review and curation. Bioinformatics 2009; 25:2271-22 78. PubMed http://dx.doi.org/10.1093/bioinformatics/btp393

13. Garrity GM, Field D, Kyrpides N, Hirschman L, Sansone SA, Anquiuoli S, Cole JR, Glöckner FO, Kolker E, Kowalshuk G, et al. Toward a standardscompliant genomic and metag enomic publication record. OMICS 2008; 12:157-160. PubMed http://dx.doi.org/10.1089/omi.2008.A2 B2
14. Garrity GM. The state of standards in genomic sciences. Stand Genomic Sci 2011; 5:262-268. PubMed http://dx.doi.org/10.4056/sigs.2515706

15. Nelson KE, Weinstock GM, Highlander SK, Worley KC, Creasy HH, Wortman JR, Rusch DB, Mitreva M, Soderg ren E, Chinwalla AT, et al. A Catalog of Reference Genomes from the Human Microbiome. Science 2010; 328:994-999. PubMed http://dx.doi.org/10.1126/science.1183605

16. Yilmaz P, Kottmann R, Field D, Knight R, Cole JR, Amaral-Zettler L, Gilbert JA, Karsch-Mizrachi I, Johnston A, Cochrane G, et al. Minimum information about a marker gene sequence (MIMARKS) and minimum information about any (x) sequence (MIXS) specifications. Nat Biotechnol 2011; 29:41 5-420. PubMed http://dx.doi.org/10.1038/nbt.1823

17. Field D, Amaral-Zettler L, Cochrane G, Cole JR, Dawyndt P, Garrity GM, Gilbert J, Glöckner FO, Hirschman L, Karsch-Mzrachi I, et al. The GenomicStandards Consortium. PLOS Biol 2011; 9:e1001 088. PubMed http://dx.doi.org/10.1371/journal.pbio. 1001088

18. Anderson I, Scheuner C, Göker M, Mavromatis K, Hooper SD, Porat I, Klenk HP, Ivanova N, Kyrpides NC. Novel Insights into the Diversity of Catabolic Metabolism from Ten Haloarchaeal Genomes. PLOS ONE 2011; 6:e20237. PubMed http://dx.doi.org/10.1371/journal.pone.0020237

19. Zhang T, Datta S, Eichler J, Ivanova N, Axen SD, Kerfeld CA, Chen F, Kyrpides NC, Hug enholtz P, Cheng JF, et al. Identification of a haloalkaliphilic and thermostable cellulase with improved ionic liquid tolerance. Green Chem (In press).

20. Anderson I, Abt B, Lykidis A, Klenk HP, Kyrpides NC, Ivanova N. Genomics of aerobic cellulose utilization systems in actinobacteria. PLOS ONE 2012; 7:e39331. PubMed http://dx.doi.org/10.1371/journal.pone.0039331 